\title{
The Design and Field Observation of a Haptic Notification System for Timing Awareness During Oral Presentations
}

\author{
Diane Tam ${ }^{1}$, Karon E. MacLean ${ }^{1}$, Joanna McGrenere ${ }^{1}$, and Katherine J. Kuchenbecker ${ }^{2}$ \\ ${ }^{1}$ University of British Columbia \\ Vancouver, BC, Canada \\ \{dianetam, maclean, joanna $\} @$ cs.ubc.ca \\ ${ }^{2}$ University of Pennsylvania \\ Philadelphia, PA, USA \\ kuchenbe@seas.upenn.edu
}

\begin{abstract}
To moderate oral presentations a chair must manage time, and communicate time parameters to speakers through a variety of means. But speakers often miss time cues, chairs cannot confirm their receipt, and the broken dialogue can be a sideshow for the audience. We developed HaNS, a wireless wrist-worn chair-speaker Haptic Notification System that delivers tactile cues for time-managing oral presentations, and performed field observations at university research seminars and two mid-sized academic conferences (input from 66 speakers, 21 chairs, and 65 audience members). Results indicate that HaNS can improve a user's awareness of time, facilitate chair-speaker coordination, and reduce distraction of speaker and audience through its private communication channel. Eliminating overruns will require improvement in speaker 'internal' control, which our results suggest HaNS can also support given practice. We conclude with design guidelines for both conference-deployed and personal timing tools, using touch or another notification modality.
\end{abstract}

\section{Author Keywords}

Oral presentation; field study; vibrotactile; wearable haptics.

\section{ACM Classification Keywords}

H.5.2. User Interfaces: Haptic I/O - Tactile \& Haptic UIs, User Interface Design, Prototyping, User-centered design.

\section{INTRODUCTION}

Early in most conferences is a plea that speakers stay on time to avoid a cascade of delays. Yet all too often a speaker abruptly realizes that time is up and ends awkwardly; or, forges into oblivious overtime - with palpable discomfort for audience, chair, and speaker.

Conferences typically depend on session chairs to manage session flow and timing. Chairs must listen to the talks and prepare their own questions, while time-managing presentation and question (Q\&A) periods. Their primary tools in this difficult job are a timer and speaker time cues (e.g., holding up a "3 minutes left" sign). However, these tools inadequately support what we call the chair-speaker loop, in which a chair issues a time cue to a speaker (loop opens) and

Permission to make digital or hard copies of all or part of this work for personal or classroom use is granted without fee provided that copies are not made or distributed for profit or commercial advantage and that copies bear this notice and the full citation on the first page. To copy otherwise, or republish, to post on servers or to redistribute to lists, requires prior specific permission and/or a fee.

CHI 2013, April 27-May 2, 2013, Paris, France.

Copyright (C) 2013 ACM 978-1-4503-1899-0/13/04...\$15.00.

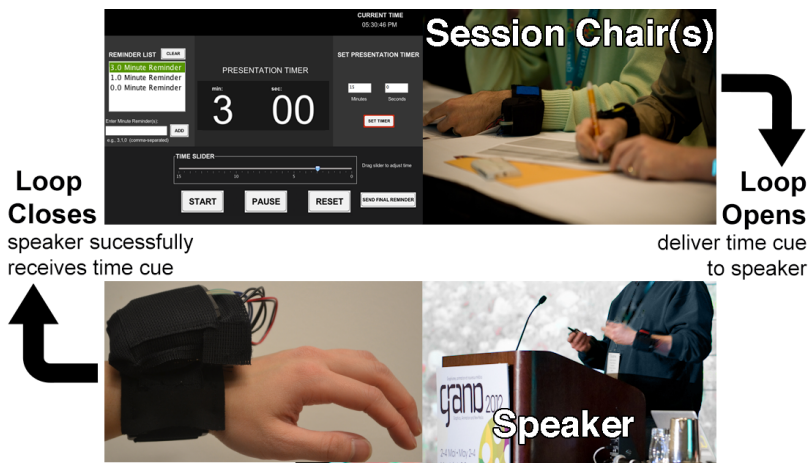

Figure 1. Haptic Notification System (HaNS) highlighting our chair-speaker loop. Left to right by row: (top) Chair's timing console; co-session chairs wearing HaNS. (bottom) Speaker wearing HaNS before; and during a presentation.

receives at minimum the speaker's acknowledgement (e.g., head nod) to confirm cue receipt (loop closes). First and foremost, the cognitive load experienced by the speaker intrudes on the communication: demands on eyes, ears, and attention easily subvert the external control administered by the chair. The speaker's internal control, although not our primary focus, is likewise undermined; this is the individual's strategic use of time awareness (gained through personal use of tools such as a wristwatch, software timer, and/or the chair's cues) to achieve effective pacing. Finally, the stress created by an audience's possible awareness of this loop can motivate a speaker to conclude the talk quickly, but also reduce his/her ability to do so. We are not aware of any tool that automates coordinated, low-cognitive-effort session timing and time awareness.

To address this void, we iteratively designed a novel wireless wrist-worn chair-speaker Haptic Notification System (HaNS) (Figure 1) that delivers tactile cues for time-managing oral presentations. We observed HaNS's deployment on chairs and speakers through field observation in numerous university research seminars and at two academic research conferences. Relative to non-HaNS timing practices, we found that HaNS can with little effort improve a wearer's awareness of the passage of time; it automatically delivers salient cues, unintrusively, privately and remotely, and it streamlines the chair-speaker loop while minimizing social implications. Unsurprisingly given our focus on external timing control, speakers using HaNS still went overtime; reducing overruns will require improving a speaker's internal control. Our results suggest HaNS may also be a powerful tool for this purpose, 
given personalization and practice. The intriguing ongoing challenge will be to optimize the sometimes-conflicting needs of institutional (e.g., conference) and personal use.

Our contributions follow: HaNS's iterative design and field evaluations, which produced subtle insights into stakeholder needs and design recommendations for a new approach to timing support, through touch or other modalities.

\section{RELATED WORK}

\section{Modality and Attention in Demanding Environments}

Theories of Managing Mental Resources. Decades of multimodal mental resource modeling make it clear that performance suffers when modalities are misused (wrong choice or too few) in conveying information [4]. Mayer's Modality Principle says for verbal-graphical information, the verbal should be presented as auditory narration since the graphical cannot [32]. Many theories (dual-channel [37], Wickens' multiple resource [48] and others) agree that humans process information more effectively when multiple channels are coordinated rather than processing all information through one, limited-capacity channel.

Attention and Interruption in Multitasking Use. Performance degradation is seen in many studies of how sensory and attentional resources interact and compete in a wide variety of task overload situations involving computation, communication, collaboration, and recovery from disruption $[3,8,47]$. Monitoring time while speaking publicly is no exception: time estimation accuracy suffers under cognitive load [39], and self-monitoring can increase self-interruption [18]. External time cues instead help provide passive awareness [18,28].

Modality-Appropriateness of Touch. Even experienced speakers can find their vision and audition saturated when continuously reading, speaking, and hearing while recalling rehearsed content stored visually and aurally, all under the overwhelming regard of the audience [30]. Maintaining time awareness is one more drain. Supporting this need under a different channel might free some audition or vision and be less distracting due to lower mental demands [13,47], while adding an information stream (time awareness) that was unavailable. Automated (pre-set) cues in any modality could theoretically privately reduce the need to poll (repeatedly check) timing parameters (e.g., auditory nonverbal clicks through an earbud device, flashing lights on the podium), but studies show that presenting information haptically reduces cognitive load on vision and audition, optimizing attention and sensory resources $[20,25,28]$. Thus, touch seems the most viable candidate from a resource standpoint in this setting, as it does not require the user to look or listen.

\section{Existing Presentation Support}

Chair-speaker presentation systems. We have found no products or studies of session timing support. The essential need is to increase speakers' and chairs' awareness of time while automating the communication loop, which existing timing systems (discussed next, mostly visual) do not address.
Commercial systems. Products that provide visual timing support to speakers (e.g., PowerPoint [31], wristwatches, smartphones, presentation remotes [26]) have evolved for decades. Some systems also support the use of silent, genericvibration alarms on phone, watch [33], or remote. The effectiveness of haptics does not appear to have been explored in the presentation context where stress levels may be high and interactions with the device must be subtle.

Research systems. Many pre-commercial concepts aim for unintrusive situational awareness of time, featuring peripheral ambient displays of light, color, and progress bars $[29,36]$ or visual displays held by the audience [10]. These are generally visual and public and again overlook chair-speaker communication. Similar trends hold in non-timing presentation-related studies such as visual content navigation methods [40], presentation control systems with visual displays [34], and presentation trainers with visual feedback [23]. Missing are non-visual awareness solutions.

\section{Tactile Perception: Body Locale and Iconography}

Body Location and Form Factor. The wrist is one of the most sensitive body locations for vibrotactile stimuli, particularly during walking and standing when lower-limb sensitivity drops, and even with visuo-cognitive distractions [22,24]. A skin-exposed location where a presentation device could be worn, the wrist is also a key site for functional social touch as observed in human-to-human [21] and device-mediated [2] interaction. Wrist-worn haptic wearables [2,6,24,41] offer insights as to construction, sensitivity, comfort, and material implications.

Design and Delivery of Meaningful Signals. Past research on haptic icon design, summarized in [27], provides a start for finding salient yet unintrusive tactile mappings for cues. Icons for progress updates, errors, and warnings provide insights for presentation cues [15,44]. Signal perception, usually vibrotactile and on the fingertips, has been examined as a function of intensity [19], duration [44], and tempo and rhythm $[12,44]$. Information can be expressively conveyed through tactile patterns [5], but at least small numbers of arbitrary mappings can be learned as easily as meaningful ones [11]. Systematic techniques are essential for larger sets $[5,44]$. For small sets, ad hoc creation with iterative testing and refinement [43] is suitable and is the approach taken here, though the diverse range of users in the presentation setting brings special challenges.

\section{HAPTIC NOTIFICATION SYSTEM (HaNS)}

HaNS has six elements that required independent and then integrated design (Figure 2): wrist-worn haptic devices to be worn by a lineup of speakers and the chair(s), the haptic cues themselves, the chair's timing console, an experimenter console for overseeing system functions, the wireless communication protocol, and finally, the numerous social protocols surrounding their use. Here we first explain process and emerging rationale, then detail the final design. 


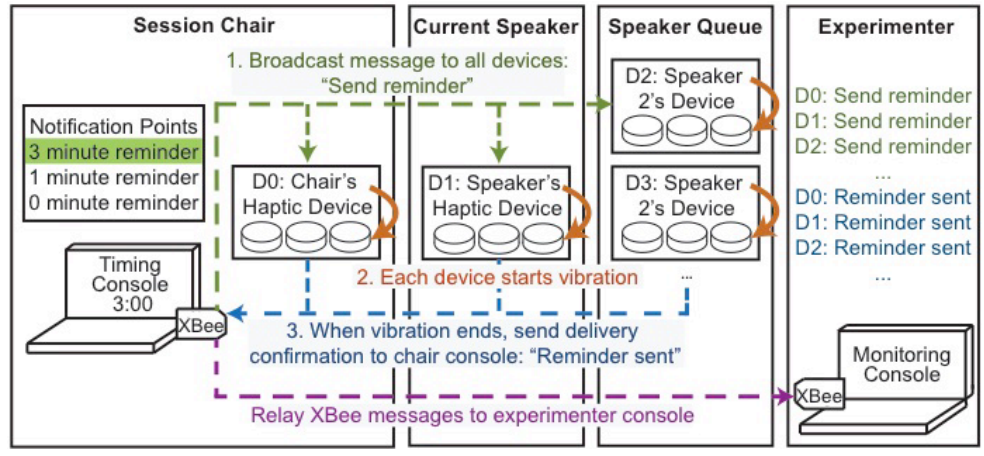

Figure 2. HaNS's system components (haptic devices, chair's timing console, experimenter console) and the wireless XBee communication flow (dashed lines) for sending a cue.

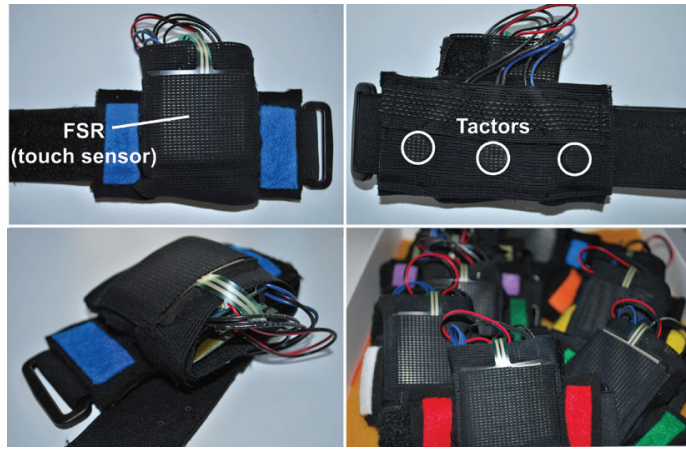

Figure 4. The haptic device. (top) Left: top view. Right: bottom view showing tactors. (bottom) Left: internal components. Right: group of haptic devices.

\section{Iterative Design and Preliminary Testing}

For a realistic yet lower-stakes development context, we invited speakers and chairs of academic seminar series to pilot-test HaNS over several months - in all, 18 presentations involving faculty, students, and guest speakers, research seminars, practice conference talks, paper discussions, and a Master's thesis defense. We interviewed 5 participants who had previously spoken at and/or chaired conference sessions for perspectives on more formal settings. Later, two mock presentation sessions (5-7 speakers and a chair) helped us further streamline communication and logistics, including efficient donning / doffing of devices. We share our key learnings here.

\section{Haptic cues: understandable and unintrusive}

We manually triggered cues during our pilots to learn which vibration patterns and which mappings to specific time points were easy for users to notice, interpret, and utilize without feeling startled. Ramping the cue, lowering vibration intensity (voltage), and using temporal patterns (Figure 3) all lessened the distractive effects of the cues while retaining much of their salience.

\section{Chair's timing console: display/trigger modes and granularity}

Our pilot chairs confirmed the value of receiving the same wristband cues as the speaker, and the need (and cognitive ability) to access detailed timing information best shown visually (we noticed chairs typically using a clock or timer). A console must also support triggering additional (unplanned) cues and canceling automated ones.

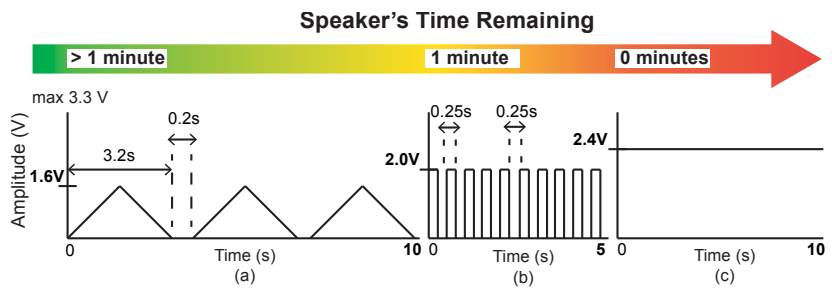

Figure 3. Haptic cues and time points for delivery: a) early warning ( $T_{r}$ (time remaining) $>1$ minute), b) urgent warning $\left(T_{r}=1 \mathrm{~m}\right)$, and c) time is up $\left(T_{r}=0 \mathrm{~m}\right)$. Vibratory frequency (under the voltage envelope) is a function of the tactor's rotational dynamics (maximum speed $\sim 12,000 \mathrm{rpm}$ ).
Chair-speaker loop: acknowledgement-free

We originally aimed simply to improve reliability of current chair-speaker communication, by delivering and confirming cues while minimizing disruption. Our largest design-phase insight was that if cues are reliably noticed, then a speaker's 'delivery receipt' (cue acknowledgement) might be unnecessary, which subtly changes the loop (Figure 1). Speakers found every explicit acknowledgement gesture we tried (head nod, eye contact, shaking or touching the wrist device) disruptive, but without exception reported noticing the cues. Thus we made cues terminate after an interval, but allowed the speaker to stop them earlier by pressing the wristband - volunteered as the most natural gesture (similar to stopping an alarm on a wristwatch), and easily detected. Upon acknowledgement, the cue concluded immediately with two quick confirmation pulses on all devices $(2.9 \mathrm{Vx} 0.1$ seconds on, $0.1 \mathrm{~s}$ off). This solution appeared to be a good compromise between chair reassurance and speaker sensory load.

\section{Description of Haptic Notification System (HaNS)}

Our final HaNS design (Figure 2) employed two or more wrist devices and a chair timing console. These components communicated wirelessly over a point-to-multipoint network (chair console to wrist devices) using XBee series 2 radios [9]. The experimenter monitored communication flow and device status (e.g., battery levels) through a custom laptop application via another XBee.

\section{System Components}

Haptic devices (Figure 4). We built 12 Velcro-attached wrist displays, each uniquely identified within the network and visually by number and colour. A flexible casing housed the following electronic components:

- Arduino Fio microcontroller [1]: Chosen for its small size, built-in XBee socket, and Lithium Polymer battery connection with a charge circuit over USB.

- XBee series 2 radio: Configured to communicate directly with the master (chair's timing console) XBee.

- Three synchronized eccentric-mass tactors (described in [22]). Multiple (more skin contact [35], redundancy), low cost tactors were spaced across the dorsal wrist at $\sim 25 \mathrm{~mm}$ intervals, parallel to the skin as in [6], and energized with pulsewidth modulated Arduino signals. 
- Touch sensor: For cue acknowledgement, a large $(45 \times 45 \mathrm{~mm})$ force sensitive resistor (FSR) on the device's outside and near-top (Figure 4) was calibrated to detect light to strong touches (min. force of $\sim 4.45 \mathrm{~N}$ ).

Chair timing console (Figure 1). A laptop Java application timed a speaker's presentation, initiated delivery of cues, and displayed time and events for the chair(s). Its XBee, the network hub, and XBee Explorer attachment enabled serial messages (e.g., "send reminder") to be sent to other XBees in the network. It had the following features:

- Countdown timer: Display of remaining speaker's time.

- Current time: Display of the current time of day.

- Timer interface: Buttons to set presentation length, start, stop, pause, resume, and reset; a slider to modify countdown time after the presentation's start.

- Cue time points setting: Entry fields for when (time remaining) cues are to be sent.

- Cue management: Automatically broadcasts cues to all haptic devices or to specific devices by ID.

- Additional overtime cue: Triggers an extra cue.

- Device cue acknowledgement on/off: Controls the user's ability to stop cues early via touch sensor.

\section{Setup and Chair-Speaker Communication Protocol}

Figure 2 shows overall system flow. Prior to the session, the chair sets the duration and cue time points. For study purposes, the experimenter enables cue acknowledgement so only the speaker can impact cue cutoff among the devices (speaker, chair(s), and speaker queue). As the presentation begins, the chair starts the countdown timer. At a preset cue time point, the chair console highlights (green flash) the respective cue and broadcasts a serial message to all devices to trigger the specified vibration (Figure 3). At vibration completion, each device automatically confirms delivery to the chair console, which then stops flashing.

\section{SYSTEM EVALUATION}

\section{Method}

We evaluated HaNS in three academic contexts over a period of three months: 1) conference research talks, 2) conference "madness" sessions, and 3) university research seminars. This diversity allowed us to study HaNS in use while varying presentation type, duration, and formality. For example, conference talks are highly visible and time-constrained, hence more formal than a small seminar. The need for organizational access led to venues in computer science and engineering, our own research areas. Upon approving our study, venue organizers provided participant recruitment contacts and assistance with on-site details.

\section{Overview of Presentation Settings}

HS: 2012 IEEE Haptics Symposium - paper \& keynote talks. One of the HaNS authors was the HS general chair, providing a unique evaluation opportunity at this 4-day, 250-attendee multidisciplinary conference on touch-interactive systems. It included six paper sessions, each with 5-7 short (8/2 minutes
talk/Q\&A) or long (12/3m) talks. Three keynote sessions had one, two, or three $30-60 \mathrm{~m}$ talks. Session co-chairs typically held up paper signs indicating the time remaining for nonHaNS speakers, and intervened if a speaker went over.

GR: 2012 GRAND - Graphics, Animation and New Media Forum - two-minute madness (2MM) talks. GRAND was a three-day annual meeting for a $\sim 65$-researcher Canadian network. Its director invited us (on short notice) to deploy HaNS in the $2 \mathrm{MM}$ where project leaders give $2 \mathrm{~m}$ freeform updates which included mostly talking and videos, but also singing and guitars. The chair managed a countdown timer projection, and at 0 s played loud rock music and cut the speaker's microphone. The highly interactive audience contributed to a live, projected Twitter feed.

SM: Seminar - university research presentations. In the University of British Columbia's CS department, we accessed four research groups (in graphics, engineering, machine learning, and $\mathrm{HCI}$ ) with regular meetings where students, faculty, and guests present research in informal updates, conference practice talks, and discussion-based brainstorming, usually structured as a $10-30 \mathrm{~m}$ talk + Q\&A period. The chair typically reminded speakers verbally and gesturally of the time as they neared their slot's end.

Haptic Cues. GR and SM speakers who used HaNS received haptic cues in addition to the setting's traditional speaker cues; HS speakers generally received one or the other. For simplicity, these occurred at: 3, 1, and 0m left (HS paper sessions, same time points as paper signs), and 60, 30, and 0s left $(G R)$. We allowed HS keynote and all SM speakers to choose their cue time points.

\section{Procedure}

In these live settings, introducing participants to HaNS, study details, and consent options required special measures, described below. Occasionally the first contact was near the session start, a point of widespread preoccupation.

Recruitment \& Compensation. To encourage participation and familiarization, we sent personalized emails to speakers and chairs with study and consent details ( $>1$ week advance for $\mathrm{HS} / \mathrm{GR},>3$ days for SM) and thanked them with a $\$ 10 \mathrm{gift}$ card (HS, SM) or reception drink ticket (GR).

Study Components. To maximize participation, participants could choose any subset of the following components:

1) Pre-study online survey: Provide background (presentation/chairing experiences and timing practices) and choose study components for participation. (Insufficient time for GR speakers to do pre-survey).

2) HaNS: Use or not use HaNS while presenting/chairing.

3) Observation: Be observed while presenting/chairing. HS and SM were video recorded; GR received manual notation by research assistants (video consent too cumbersome, in part given short lead time).

4) Post-study online survey: Provide feedback about experience. (The GR survey used a question subset). 


\begin{tabular}{|c|c|c|c|c|c|c|c|}
\hline Setting & Participant & Pool Size & HaNS? & Total \# Participated & Pre-study & Observation & Post-study \\
\hline $\begin{array}{l}\text { Haptics } \\
\text { Symposium } \\
\text { (HS) }\end{array}$ & $\begin{array}{l}\text { Speaker }\left(\mathrm{HS}_{\mathrm{spk}}\right) \\
\text { Session chair }\left(\mathrm{HS}_{\text {chair }}\right) \\
\text { Audience }\left(\mathrm{HS}_{\mathrm{aud}}\right)\end{array}$ & $\begin{array}{r}42 \\
16 \\
250 \\
\end{array}$ & $\begin{array}{c}\mathbf{h} / n h \\
\mathbf{h} \\
- \\
\end{array}$ & $\begin{array}{c}22 \text { (1 female }) / 6 \\
15 \text { (6 female }) \\
50 \\
\end{array}$ & $\begin{array}{c}21 / 6 \\
15 \\
- \\
\end{array}$ & $\begin{array}{c}19 / 3 \\
11 \\
- \\
\end{array}$ & $\begin{array}{c}21 / 4 \\
14 \\
50 \\
\end{array}$ \\
\hline $\begin{array}{l}\text { GRAND } \\
(\mathrm{GR})\end{array}$ & $\begin{array}{l}\text { Speaker }\left(\mathrm{GR}_{\mathrm{spk}}\right) \\
\text { Session chair }\left(\mathrm{GR}_{\text {chair }}\right)\end{array}$ & $\begin{array}{c}30 \\
1 \\
\end{array}$ & $\begin{array}{c}\mathbf{h} / n h \\
\mathbf{h} \\
\end{array}$ & $\begin{array}{c}24 \text { (10 female) / } 3 \text { (2) } \\
1\end{array}$ & $\begin{array}{l}- \\
1 \\
\end{array}$ & $\begin{array}{c}24 / 2 \\
1 \\
\end{array}$ & $\begin{array}{c}22 / 2 \\
1 \\
\end{array}$ \\
\hline $\begin{array}{l}\text { Seminar } \\
\text { (SM) }\end{array}$ & $\begin{array}{l}\text { Speaker }\left(\mathrm{SM}_{\mathrm{spk}}\right) \\
\text { Session chair }\left(\mathrm{SM}_{\text {chair }}\right) \\
\text { Audience }\left(\mathrm{SM}_{\mathrm{aud}}\right)\end{array}$ & $\begin{array}{c}11 \\
5 \\
\sim 50 \text { unique } \\
\end{array}$ & $\begin{array}{l}\mathbf{h} \\
\mathbf{h} \\
- \\
\end{array}$ & $\begin{array}{c}11 \text { (2 female) } \\
5 \\
15 \\
\end{array}$ & $\begin{array}{c}11 \\
5 \\
- \\
\end{array}$ & $\begin{array}{c}11 \\
5 \\
- \\
\end{array}$ & $\begin{array}{c}11 \\
5 \\
15 \\
\end{array}$ \\
\hline TOTAL & $\begin{array}{l}\text { Speaker } \\
\text { Session chair } \\
\text { Audience }\end{array}$ & $\begin{array}{c}83 \\
22 \\
-\end{array}$ & $\begin{array}{c}\mathbf{h} / n h \\
\mathbf{h} \\
-\end{array}$ & $\begin{array}{c}57 / 9 \\
21 \\
65\end{array}$ & $\begin{array}{c}32 / 6 \\
21 \\
-\end{array}$ & $\begin{array}{c}54 / 5 \\
17 \\
-\end{array}$ & $\begin{array}{c}54 / 6 \\
20 \\
65\end{array}$ \\
\hline
\end{tabular}

Table 1. Participation counts for each study component, using (h) or not using $(n h)$ HaNS, across settings.

The speaker/chair surveys [42] asked 28-64 questions (audience survey: $\sim 12$ ). We triangulated responses from a series of multiple-choice (e.g., What do you like about the haptic device?), Likert - strongly dis/agree (e.g., The haptic reminders allowed me to focus on the presentations without monitoring the time myself), and freeform questions (e.g., How would you improve the haptic device?).

Advance Familiarization and Consent Signing. Participants could try HaNS and discuss the study before giving consent at demo sessions or just before their sessions started.

Session flow. Prior to a session, HaNS's timing console was set up on the chair's table (the experimenter controlled HaNS's timer for GR). Queued HaNS participants were given a device to wear on their chosen wrist, in advance to prevent disruptions and to allow speakers to experience others' cues. At the GR 2MM where speakers outnumbered devices, speakers coming offstage handed theirs into the queue. Postsession, participants received a thank-you letter with a link to the post-survey and compensation if given.

\section{Participant Summary}

Speaker and session chair participants (Table 1) included graduate students, postdocs, faculty, and industry researchers. There was some participation overlap amongst HaNS wearers: 7 (4 SM, 3 HS) speakers had been pilots, 1 (HS) presented twice, and 3 (HS) speakers also participated as chairs. However, HaNS had evolved significantly since early piloting, and presentation type, length, venue, audience, and/or role varied, so the experience remained quite novel even for these users. HS and SM audience members were also invited to complete a post-study survey regarding their overall impression of presentation timing.

\section{Results}

Data was collected from 59 pre- and 145 post-surveys, 22 hours of video, and 27 manual speaker/chair observations. 78 participants wore the haptic device (46/32 on left/right wrist, without correlation to hand dominance or watch-wearing). We analyzed quantitative data descriptively and qualitative data by top-down content analysis open-coding [7], triangulating our multiple sources. Observed and self-reported experiences with current timing methods were unsurprising, so we share only their highlights. We then report on users' HaNS experience as a) impact on overall presentation time, b) feasibility as a conference timing system, and emergent c) improvements and d) limitations.

\section{Current Practice Highlights}

The following highlights data from pre-survey reports with supporting post-survey and observations.

Speaker Cues. Among the participants who reported receiving/delivering speaker cues before, most speakers (HS:79\% [15/19], SM:100\% [10/10], GR:-) and chairs (HS:71\% [10/14], SM:40\% [2/5], GR:-) reported experience with visual sign cues, also noting a chair's gestures, standing up, verbal cues, or ringing a bell. Some speakers found chair cues easy to notice (HS:68\% [13/19], SM:30\% [3/10], GR:-). However, HS chairs were observed holding the sign (at varying heights) for $5 \mathrm{~s}$ (avg; $\max 25$ ) before the speaker seemed to notice. All $11 \mathrm{HS}$ chairs who used signs during the study reported wanting speaker acknowledgement: "[The speakers] are so focused - they do not see the [paper] signs" $\left[\mathrm{HS}_{\text {chair }} 1\right]$. HS sign delivery was also delayed by $4 \mathrm{~s}$ (avg; max 12); the chairs varied in how fast they picked up the sign. Chairs generally disliked the responsibility of interrupting speakers (HS:57\% [8/14], SM:80\% [4/5], GR:-), and 5 (HS) explicitly wished timing was communicated automatically.

Most speakers (HS:63\% [12/19], SM:60\% [6/10], GR:-) and chairs (HS:64\% [9/14], SM:60\% [3/5], GR:-) disliked the audience noticing speaker cues. 76\% [38/50] of HS audience respondents reported noticing or being distracted by the signs: "I was watching the signs and waiting to see when the speaker would notice it. Shared anticipation...It's distracting" [HS $\left.{ }_{\text {aud }} 15\right]$. Some speakers also found visual signs distracting (HS:32\% [6/19], SM:20\% [2/10], GR:-). At HS, we noticed speakers glancing at the sign, nodding to the chair, commenting (e.g., $\mathrm{HS}_{\mathrm{spk}} 16$ said "Ok" to the chair who missed his head nod), and/or pausing or stumbling in their speech upon seeing the sign.

Personal Timing Tools. Few speakers (HS:31\% [4/13], SM:25\% [2/8], GR:-) and chairs (HS:36\% [5/14], SM:0\% 
[0/5], GR:-) who reported regular use of a timing tool (usually a clock or cell/smartphone stopwatch) felt it was easy to notice the time on their tools while presenting or chairing. All speakers and $84 \%$ [16/19] of these chairs felt it effortful to continually poll the time. Visibility and time starting were cited as problems: "I can't prop [the cellphone] up to face me, it lays flat on the table / lectern in front of me" $\left[\mathrm{SM}_{\mathrm{spk}} 6\right]$ and "Sometimes I forget to start [the software timer]" $\left[\mathrm{HS}_{\mathrm{spk}} 21\right]$.

HaNS - Impact on Presentation Outcome

HS and SM speakers (GR 2MM did not permit overtime) ended late irrespective of HaNS's use: HaNS - HS:43\% [10/23] (avg 51s over), SM:55\% [6/11] (77s); non-HaNS HS:45\% [9/20] (32s). Among the overtime HaNS speakers, 4 (3 HS, 1 SM) were invited keynote or guest presenters who had been instructed to take their needed time. 1 (HS) keynote and 2 other (HS) speakers had technical difficulties, and 1 (SM) took questions while presenting. 6 (HS) chairs also tended to start the timer slightly early, further cutting into a talk's time. None of the non-HaNS speakers went overtime for the above reasons. Of the remainder, 5 (3 HS, $2 \mathrm{SM}$ ) quickly concluded their talks upon receiving the final $0 \mathrm{~m}$ cue, while 4 (2 HS, $2 \mathrm{SM}$ ) others continued to talk even when aware of the time. The most overtime speaker said 'I'm just about out of time, but I'm almost at the end" [ $\left.\mathrm{SM}_{\mathrm{spk}} 9\right]$, then spoke for another $2: 41 \mathrm{~m}$.

Emerging here is a distinction between time awareness and strategic use of that information. Most speakers stated in the pre-survey that it was important to finish their material (HS:88\% [22/25], SM:91\% [10/11], GR:-). But awareness alone may not alter behavior: "People who went over their time despite the haptic reminders probably chose to ignore it" [HS $\left.\mathrm{Hud}_{\mathrm{aud}} 36\right]$. 5 GR speakers epitomized this commitment, battling on amidst blaring music and sans microphone.

HaNS - Feasibility as a Chair-Speaker Timing System We analyzed post-survey responses (abridged for GR) and observations for HaNS's impact on parameters highlighted earlier as crucial yet lacking in existing systems.

Cues were salient. Most speakers (HS:90\% [19/21], SM:73\% [8/11], GR:68\% [15/22]) and all chairs reported HaNS's cues were easy to notice. Chairs also thought the cues to be noticeable for speakers (HS:93\% [13/14], SM:100\% [5/5], GR:-) such that few felt explicit speaker acknowledgement was necessary (HS:29\% [4/14], SM:40\% [2/5], GR:-): "I was confident that the speakers noticed [HaNS's cues] right away" $\left[\mathrm{HS}_{\text {chair }} 15\right]$.

Cues were private and minimally captured attention. Few audience members noticed HaNS cue delivery (HS:14\% [7/50], SM:27\% [4/15], GR:-). The only apparent clues were explicit speaker comments - "I'm getting buzzed!" - or exaggerated gestures (slapping the device). "No. I couldn't notice [HaNS's cues]. A few speakers mentioned they were being buzzed, but I think they did so mostly to be funny" $\left[\mathrm{HS}_{\text {aud }} 19\right]$. Otherwise, there were no external signs of speakers startling or breaking their speech due to the cues. Few speakers (HS:24\% [5/21], SM:27\% [3/11], GR:18\% [4/22]) and only $1 \mathrm{HS}$ chair reported the cues to be distracting. Attendees also appreciated the subtleties: "The adherence to schedule was high without the normal 'verbal harassment'...great as a conference attendee" [HS $\left.{ }_{\mathrm{aud}} 9\right]$, "[HaNS's cues] are unobtrusive to the audience yet noticeable to the speaker without them having to look and respond" [ $\left.\mathrm{HS}_{\mathrm{aud}} 26\right]$, and "I liked that as an audience I wasn't involved in the timing issues of the presenter" [ $\left.\mathrm{SM}_{\mathrm{aud}} 9\right]$.

Automation (and precision) improved. HaNS delivered cues promptly and consistently, and was thus very accurate. Chairs liked how they were automatically notified of the time (HS:93\% [13/14], SM:100\% [5/5], GR:100\% [1/1]) and relieved of delivering cues (HS:71\% [10/14], SM:100\% [5/5], GR:-). Most speakers also found the automation helpful, but (notably for GR) were less enthused (HS:71\% [15/21], SM:64\% [7/11], GR:55\% [12/22]).

Chair-speaker channel was viable and utilized. Most chairs (HS:93\% [13/14], SM:100\% [5/5], GR:-) felt HaNS helped with their chairing duties; $91 \%$ [10/11] of the HS chairs who both wore the haptic device and delivered visual signs felt the device was better at reminding speakers of the time. Other chair-speaker uses were noted. 1 (HS) speaker and 1 (SM) chair wanted cues to communicate when the chair has started the timer. 2 (HS) chairs used the timing console's overtime button to send extra cues to overtime speakers: "When one speaker went over I buzzed him again and it was clear that he really got the message!" [ $\left.\mathrm{HS}_{\text {chair }} 14\right]$ This function was flagged as useful in the Q\&A period: "I wonder if the session chairs could give the speaker a little buzz when s/he is too longwinded in answering a question" [ $\left[\mathrm{HS}_{\mathrm{aud}} 21\right]$.

HaNS cues created opportunities for improved timing. We observed speakers' behavior changes after a HaNS cue and unsurprisingly, witnessed speakers adjusting their pace (e.g., talking faster or slower, skipping slides). At least 8 (HS) even mentioned timing, e.g., "I noticed I'm a bit fast" [ $\left[\mathrm{HS}_{\mathrm{spk}} 14\right]$ and "I'm going to have to move through this quickly" [HS $\left.{ }_{\mathrm{spk}} 19\right] .14$ (3 HS, $\left.10 \mathrm{SM}, 1 \mathrm{GR}\right)$ noted that HaNS allowed them to decide whether the talk was going too fast or too slow. Upon receiving the $3 \mathrm{~m}$ cue, $\mathrm{HS}_{\mathrm{spk}} 14$ was on his conclusion slides. He mentioned that he was a bit fast, then took $2: 16 \mathrm{~m}$ to elaborately summarize. $\mathrm{HS}_{\mathrm{spk}} 16$ prepared a $15 \mathrm{~m}$ talk, discovered the length error at the session start, but selectively skipped content and finished only 24 s overtime. 14 (7 HS, 5 SM, 2 GR) also noted the halfway cue as particularly useful for gauging overall progress: "Based on the half-point reminder, I realized I was behind and I therefore left out some

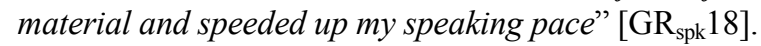

Users saw value for HaNS internal' presentation control. Many speakers (HS:81\% [17/21], SM:82\% [9/11], GR:-) and chairs (HS:86\% [12/14]; SM:100\% [5/5], GR:-) indicated satisfaction with HaNS's cues in helping them track time. Again, notably more for HS and SM than GR, many speakers (HS:86\% [18/21], SM:82\% [9/11], GR:50\% [11/22]) and chairs (HS:93\% [13/14], SM:100\% [5/5], GR:100\% [1/1]) 
also agreed the device would be useful in a future presentation or session. Most chairs felt the device was better than their personal timing tools for tracking speakers' time (HS:86\% [12/14], SM:100\% [5/5], GR:100\% [1/1]), as did about half of speakers (HS:67\% [14/21], SM:36\% [4/11], GR:41\% [9/22]). HaNS seems to be appreciated broadly, but somewhat more by chairs than speakers, and by HS and SM speakers than GR.

\section{HaNS - Improvements Based on User Feedback}

Responses from and observations of users and audience members indicated ways in which HaNS can be improved.

Full utility of cues may take practice. While many chairs found the cues easy to understand (HS:79\% [11/14], SM:80\% [4/5], GR:100\% [1/1]), fewer, yet still the majority of speakers agreed (HS:76\% [16/21], SM:45\% [5/11], GR:59\% [13/22]). Speakers reported using both vibration pattern (HS:57\% [12/21], SM:64\% [7/11], GR:-) and sequence (HS:71\% [15/21], SM:64\% [7/11], GR:-) to distinguish cues. However, learning the mapping may require multiple exposures: "With a couple more uses the understandability would have been no problem at all” [ $\left[\mathrm{GR}_{\mathrm{spk}} 18\right]$.

Wearability allowed freedom of movement but physical device should be smaller and more versatile. HaNS's wearability untethered users: "Having [the device] on my wrist allows me to walk around the presentation space" [ $\left.\mathrm{SM}_{\mathrm{spk}} 9\right]$, and "During the Q\&A session I left the chairing table where the laptop with timing information was...it was possible for me to gracefully end the $Q \& A$ session without glancing at my watch or run back to the chairing table" $\left[\mathrm{HS}_{\mathrm{chair}} 2\right]$. However, designed for robustness, our devices were larger than ideal, as noted by $43 \%$ [23/54]/55\% [11/20] of all speakers/chairs. 4 speakers (1 SM, $3 \mathrm{GR}$ ) and 4 chairs (2 HS, 2 SM) also felt such a system should be wearable at multiple locations or even handheld: "I put [the device] in my pocket, on my ankle, I sat on it and strapped it to my belt" $\left[\mathrm{HS}_{\mathrm{chair}} 9\right]$.

Cue acknowledgement is visible and sometimes missed. Acknowledgement was optional, but some speakers touched the device sensor to stop the vibrations (HS:24\% [5/21], SM:27\% [3/11], GR:-). This was externally noticeable, especially with an exaggerated gesture. Our FSR sensor also missed light touches by 4 (HS) speakers, e.g., quick brushes by nervous speakers. Exaggerated gestures may have been precautions taken against misses. 3 (HS) chairs reported also wanting acknowledgement privileges for their own device.

Advanced timing support. This version of HaNS could time a single instance, e.g., one presentation. 9 HS chairs found it cumbersome to reconfigure the timer for each speaker, especially for varied talk lengths. Other intervals (total session, Q\&A, introductions, conclusions and thank yous, between-talk transitions) were also unaccounted for. Chairs constantly re-evaluate progress to stay on schedule: " $A$ session chair has to solve a constraint satisfaction problem in the way that is the most 'fair' to the speakers and audience. The $Q \& A$ will typically not be of fixed duration, hence, use the session chair's judgment" [ $\left.\mathrm{SM}_{\text {chair }} 4\right]$.
HaNS - Limitations of external speaker cues

HaNS is modeled after existing speaker cues, which focus solely on 'external' speaker management. We discovered several limitations that are likely to be common to any such scheme that does not incorporate speakers' need to internally strategize pacing and thereby avoid overruns.

Practice is needed to effectively utilize cues. Many SM but fewer HS speakers felt satisfied with the overall pace of their presentations (HS:52\% [11/21], SM:82\% [9/11], GR:-). Speakers only had a brief demo of HaNS and did not rehearse with the cues. Additional practice might allow speakers to better utilize an improved time awareness: "the device makes sense only if the presenter had also done his practice with it" $\left[\mathrm{SM}_{\mathrm{spk}} 11\right]$ and "I would need to have practiced with [HaNS's cues] for them to have been useful” [ $\left[\mathrm{GR}_{\mathrm{spk}} 23\right]$.

Choosing appropriate cues requires familiarity. About half of speakers suggested different cue time points for a future presentation (HS:48\% [12/25], SM:45\% [5/11], GR:-). But 8 (3 HS, 2 SM, 3 GR) again noted the key role of practice: "The halfway reminder was the most useful because I didn't really have any sub-timings practiced" [ $\left.\mathrm{SM}_{\mathrm{spk}} 9\right]$ and "If I had timed my presentation during my practice, i.e., I knew exactly how many minutes should be left at each slide, then I could probably choose more meaningful reminders" [ $\left.\mathrm{SM}_{\mathrm{spk}} 11\right]$.

Fine-grained timing must also be available. Discrete cues help one check pace at particular points. However, many HaNS speakers unsurprisingly reported still needing access to the time in between cues (HS:43\% [9/21], SM:18\% [2/11], GR:55\% [12/22]). In fact, a few HaNS speakers additionally used a personal timing tool (HS:10\% [2/21], SM:27\% [3/11], GR:-). We noticed speakers checking these while presenting, and glancing at the haptic device as if it was a watch. 7 GR speakers reported relying on the conference-provided visual timer in front of them: "I relied heavily on the digital clock in front of me, which allowed me to time my talk down to the last second" [ $\left[\mathrm{GR}_{\mathrm{spk}} 22\right]$. 11 speakers (2 HS, $\left.4 \mathrm{SM}, 5 \mathrm{GR}\right)$ and 3 (HS) chairs suggested the device be used with a visual clock.

Delivering cues according to progress. HaNS sent cues regardless of a speaker's progress. 5 speakers (3 HS, 2 GR) and 6 chairs (5 HS, $1 \mathrm{SM}$ ) suggested that a timing system should gauge progress for you to effectively pace, e.g., slow down, speed up talk: "In some scenarios, it may be more important to know whether you are ahead-of-time or behindtime at a particular point in a presentation, rather than knowing that there is one minute left" $\left[\mathrm{GR}_{\mathrm{spk}} 14\right]$. Further, speakers may want to receive cues only when they are behind: "[the visual sign] often appears at a point where I am clearly wrapping up the talk...If I'm running late and almost out of time give me a strong reminder. If I'm doing fine leave me alone!" $\left[\mathrm{HS}_{\mathrm{spk}} 1\right]$

Importance of personalization. Participants' perceptions varied in how HaNS's cues should be improved. For example, 6 GR speakers reported noticing the cues while queued, but not while presenting. With HaNS, the cues can theoretically 
be customized (e.g., made stronger), whereas a speaker cannot reasonably personalize how a chair delivers cues. In total, 7 speakers (1 HS, 3 SM, $3 \mathrm{GR}$ ) wanted stronger vibrations, while 2 (HS) wanted them weaker. 4 speakers (2 HS, 2 GR) and 3 (HS) chairs felt cues were too long or short, or that there were too many signals. 16 speakers ( 7 HS, 4 SM, 5 GR) and 6 chairs (5 HS, $1 \mathrm{GR}$ ) also suggested alternative haptic mappings (14 preferred different signals and 2 all the same).

\section{DISCUSSION}

The diversity of our evaluation settings (formality, length, and type) afforded a glimpse of where HaNS has immediate value and how it works with users in contrast to traditional methods.

HaNS executes two primary operations to facilitate chairspeaker communication: timing automation, wherein timing management is offloaded from the chair, and reliable and private (because they are haptic) cue delivery. Although each could theoretically provide benefits alone, our particular interest is in the sensory, cognitive, and social outcomes of their interplay and context. For example, to the extent that HaNS cues intrude, they may with continued use become more comfortable because (due to automation) they can be highly consistent, unlike manual systems that vary in delivery and receipt. Similarly, the speaker's workload benefit due to optional acknowledgement is only available when the chair can assume confirmed on-time delivery of salient signals (requiring both HaNS operations).

Furthermore, one of our primary insights pertains to external versus internal loci of speaker timing control, and how HaNS (automation and notification) might also be well suited for internal control. In essence, slicing HaNS operations a different way may facilitate a system-speaker additionally to chair-speaker loop, personalized if possible.

These themes guide our reflections on what we have learned about touch-based time cues and speaker control.

\section{Haptic Modality}

The need that triggered this research was for salient, unintrusive cues that can be delivered privately and remotely, a standard which HaNS cues appear to meet. We have further identified benefits of automating delivery and potentially, of personalizing timing, signal content, and other cue behaviors. Validation with other modalities is needed, but inherent attributes of touch (as laid out below and in the Related Work) make it ideal for this context. However, its optimal design is not without subtleties.

Individuals and contexts vary in perceptual capacity. People's differing sensitivity to touch, accentuated by context and cognitive load [22], could explain why a few participants (notably GR) found the cues hard to notice. Tactile sensitivity also drops with movement [38], impacting mobile speakers particularly. Cognitive theories agree that any perceptual modality will 'miss' under extreme sensory loads, so it is understandable that in the frankly chaotic GR setting, fewer speakers noticed HaNS cues. Together these point to both personal and contextual tuning of signal loudness, to maximize the population that can benefit.

Novices can process simple haptic information immediately; potential for expert usage with training. Unlike speakers, chairs could easily visually refer to the timing console to distinguish the cues. Most HS and SM speakers were relying on HaNS's cues alone (only 16\% [5/32] also used a personal timing tool). While some of our speakers had familiarization time, few got to really practice using HaNS cues, which is typical of speaker cues. Nevertheless, most seemed to easily find adequate mnemonics based on a combination of order and signal characteristics; the current signal mapping alone was not generally enough. However, humans can process tactile information while under high cognitive load [5,43], and with training, are able to parse haptic signals more proficiently [45]. Also, the variety of signals preferred by 14 users suggests that personalized cues may allow for more intuitive processing. There is thus reason to expect that with training this already promising performance standard might rise, further lowering mental workload and signal utility.

Social factors generally improved. Most chairs valued relief from the social responsibility for delivering and confirming receipt of speaker cues that accrued from HaNS's automation and privacy. We also wondered if, when a speaker's time remaining is not publically broadcasted, reduced social pressure would allow speakers to conclude more elegantly, or conversely, be less mindful of finishing on time. We found that speakers do generally value the importance of presentation timing, and audience members prefer not to be distracted by a speaker's timing issues; however, actual timing performance probably requires more practice to improve. In cases where speakers do need additional social pressure, chairs are still free to supply it with additional, more persistent or more public means.

\section{Inherent Nature of External Speaker Control}

HaNS was initially envisioned as a reliable mechanism for chairs to police speakers. In retrospect, it is not surprising that on average a greater percentage of chairs were positive relative to speakers, although many speakers found value as well. We offer three key explanations. First, speakers, who are in a higher stress situation than chairs, may prefer not to be controlled (will go over regardless of the time). While HaNS was intended to improve speakers' awareness of time relative to traditional methods (e.g., visual signs), it is still a form of control. The second and third reasons relate to what speakers likely need to facilitate their internal control, namely affordances and personalization, as described next.

A lower fraction of all speakers reported a preference for HaNS over their usual timing tools $(50 \%$ [27/54]) or an expectation to rely on it alone (43\% [23/54]). While HaNS offers some unique affordances to speakers (e.g., ability to leave the podium, not reliant on visual attention), it also lacks some key affordances of personal timing tools, most notably access to fine-grained timing information. This was an issue for some speakers, particularly GR; the granularity of seconds 
in between cues was important. Cue delivery may also occur at inopportune or unnecessary points. In a manual system, speakers will check the time when cognitively able to, and chairs may delay delivery until a pause occurs or choose not to deliver a cue if the speaker is almost done. Breakpoint detection research offers further insights into how cues might be managed in an automated system [16,17]. However, immediate delivery appears acceptable as message urgency increases [46]; more generally, matching the attentional draw of a cue based to its level of urgency has shown promise [14].

It is likely that the very individual strategies that speakers use mean that cue timing, signal content, and loudness need to be personalized, and practiced. Even after a single use, many speakers preferred different cue time points, signal mappings, and other customizations. Varied speaker ratings across the settings (notably GR but on occasion HS) may imply contextand presentation-type-specific modes are also needed. HaNS has many personalization opportunities: anecdotally, colleagues who have informally used HaNS several times and with customization mention increasing ability to learn how to make use of it. One can even imagine that extremely short talks with down-to-the-second timing could be an ideal use case for a practiced, attuned user.

\section{IMPLICATIONS FOR HaNS DESIGN}

We close by itemizing our requirements for jointly optimizing the apparently conflicting needs of a chair and a speaker. This list merges starting requirements that our evaluation has now confirmed, and newly emerged ones. It begins with the more demanding needs of the speaker, many aspects of which the chair shares, then adds criteria that apply to the chair alone.

\section{Speaker}

Above all, must be easy and automatic. Cues should be easy to notice and understand, yet impose minimal distraction, cognitive disruption or startle effect. Even small amounts of practice should lead to non-conscious signal processing. Setup and training must require almost zero effort.

Acknowledgement should be optional. If used, it must be a quick, easy, private motion yet immune to accidents, and include confirmatory feedback.

Develop avenues for speakers to personalize and practice, even in a conference setting. Settings for cue time points, intensity, duration, and signal mapping could be part of a user profile, set up online in advance during a familiarization session. As tactile cues become standard practice, on-site rehearsal may become unnecessary while personalized settings become imperative.

Private is better. Minimize audience awareness of cues, physical wearable, and any acknowledgement.

Coordinate detailed visual backup. More fine-grained timing information should complement tactile cues.

Must be wearable, wireless, and comfortable. Should flexibly accommodate a diversity of presentation settings and body sizes, and never impede movement. Signals need to be perceptible even with movement, and the unit itself must be accessible for any required manual interaction.

Reliability is essential. All aspects of the device must be failsafe - users must be able to absolutely rely on it.

\section{Chair}

Echo the speaker's experience. Send the same signals to the chair as the speaker, for the chair's time awareness and to replace lost external signs of the communication loop. Allow the chair to stop only his/her own cues early if needed.

Provide an additional direct, yet private communication channel. Allow the chair to provide additional cues, and other communicative elements (e.g., timer start, Q\&A is over). Also allow the chair to escalate communication to apply social pressure when needed (e.g., cue acknowledgment feature).

Make the timing system setup seamless and flexible. Chairs want to set up a whole session, including the Q\&A, to easily adjust timing as the event proceeds, and monitor progress against larger timing targets.

\section{LIMITATIONS}

We felt it crucial to test HaNS with real speaker/chair stressors and goals, and thus embarked on this ambitious study 'in the wild' where we had limited control. This did constrain our conclusions. It is also hard to know if our participants in haptics and new media were more receptive towards this intervention than others would be, or more critical given expertise and a concern for 'getting it right'. Controlled realistic tests will not become easier; voluntary uptake may be the best future indicator of usability.

\section{CONCLUSIONS AND FUTURE WORK}

HaNS, a novel wireless, wrist-worn chair-speaker communication system, uses automation and tactile cues to facilitate the time-managing of oral presentations. Through field observation we found that HaNS positively modifies the chair-speaker loop, even to the point of making speaker confirmation optional. It achieves this through reliable cue delivery, cognitive offloading, and channel privacy. HaNS can also improve time awareness. Future studies including other modalities would help further triangulate these results.

A deeper look at the inherent mismatch between traditional external speaker control and more effective speaker internal control leads us to consider how HaNS could allow both players to manage their own experience. In particular, a speaker who personalizes and practices with the cue system may be better able to use cue-derived time awareness to actually improve talk timing. Early feedback from our ongoing longitudinal observations of HaNS in a classroom lecture setting suggests this to be the case.

Finally, we look forward to watching how the current chairspeaker paradigm shifts over time as automatic and/or tactile systems like HaNS become widely adopted personal and shared tools; how timing needs range far beyond oral presentation, and evolving our own design in response. 


\section{ACKNOWLEDGMENTS}

We thank members of Haptics Symposium, GRAND, and UBC who participated in this research. This work was funded by NSERC and the GRAND NCE.

\section{REFERENCES}

1. Arduino. 2012. http://arduino.cc/en/Main/ArduinoBoardFio.

2. Baumann, M.A., MacLean, K.E., Hazelton, T.W., and McKay, A. Emulating human attention-getting practices with wearable haptics. Proc. HAPTICS, IEEE (2010), 149-156.

3. Brehmer, M., McGrenere, J., Tang, C., and Jacova, C. Effects of interruptions on older adults' computerised cognitive testing performance. Proc. CHI, ACM (2012), 2649-2658.

4. Cao, Y., Theune, M., and Nijholt, A. Towards cognitive-aware multimodal presentation: The modality effects in high-load HCI. Proc. EPCE, Springer-Verlag (2009), 3-12.

5. Chan, A., MacLean, K., and McGrenere, J. Designing haptic icons to support collaborative turn-taking. Int. J. Hum.-Comput. Stud. 66, 5 (2008), 333-355.

6. Chen, H., Santos, J., Graves, M., Kim, K., and Tan, H.Z. Tactor localization at the wrist. Proc. EuroHaptics, Springer-Verlag (2008), 209-218.

7. Corbin, J. and Strauss, A. Basics of qualitative research. Sage Publications, CA, USA, 2008.

8. Czerwinski, M., Horvitz, E., and Wilhite, S. A diary study of task switching and interruptions. Proc. CHI, ACM (2004), 175-182.

9. Digi. 2012. http://www.digi.com/xbee/.

10. Dredge, S. MIT's Dr Joseph Paradiso talks Smart Spaces, 2007. http://www.techdigest.tv/2007/12/mits_dr_joseph.html.

11. Enriquez, M. and MacLean, K. The role of choice in longitudinal recall of meaningful tactile signals. Proc. HAPTICS, IEEE (2008), 49-56.

12. Van Erp, J. and Spapé, M. Distilling the underlying dimensions of tactile melodies. Proc. EuroHaptics, (2003), 111-120.

13. Gillie, T. and Broadbent, D. What makes interruptions disruptive? A study of length, similarity, and complexity. Psychological Research 50, 4 (1989), 243-250.

14. Gluck, J., Bunt, A., and McGrenere, J. Matching attentional draw with utility in interruption. Proc. CHI, ACM (2007), 41-50.

15. Hoggan, E., Raisamo, R., and Brewster, S.A. Mapping information to audio and tactile icons. Proc. ICMI-MLMI, ACM (2009), 327-334.

16. Iqbal, S.T. and Bailey, B.P. Effects of intelligent notification management on users and their tasks. Proc. CHI, ACM (2008), 93-102.

17. Iqbal, S.T. and Bailey, B.P. Oasis: A framework for linking notification delivery to the perceptual structure of goal-directed tasks. ACM TOCHI 17, 4 (2010), 15:1-15:28.

18. Iqbal, S.T. and Horvitz, E. Notifications and awareness: a field study of alert usage and preferences. Proc. CSCW, ACM (2010), 27-30.

19. Israr, A., Tan, H.Z., and Reed, C.M. Frequency and amplitude discrimination along the kinesthetic-cutaneous continuum in the presence of masking stimuli. J. Acoust. Soc. Am. 120, 5 Pt 1 (2006), 2789-2800.

20. Jones, M.G., Minogue, J., Tretter, T.R., Negishi, A., and Taylor, R. Haptic augmentation of science instruction: Does touch matter? Science Education 90, 1 (2006), 111-123.

21. Jones, S.E. and Yarbrough, A.E. A naturalistic study of the meanings of touch. Comm. Monographs 52, 1 (1985), 19-56.

22. Karuei, I., MacLean, K.E., Foley-Fisher, Z., MacKenzie, R., Koch, S., and El-Zohairy, M. Detecting vibrations across the body in mobile contexts. Proc. CHI, ACM (2011), 3267-3276.
23. Kurihara, K., Goto, M., Ogata, J., Matsusaka, Y., and Igarashi, T. Presentation sensei. Proc. ICMI, ACM (2007), 358-365.

24. Lee, S.C. and Starner, T. BuzzWear: alert perception in wearable tactile displays on the wrist. Proc. CHI, ACM (2010), 433-442.

25. Leung, R., MacLean, K., Bertelsen, M.B., and Saubhasik, M. Evaluation of haptically augmented touchscreen GUI elements under cognitive load. Proc. ICMI, ACM (2007), 374-381.

26. Logitech. 2012. http://www.logitech.com/en-ca/remotes.

27. MacLean, K.E. Foundations of transparency in tactile information design. IEEE Trans. on Haptics 1, 2 (2008), 84 -95.

28. MacLean, K.E. Putting haptics into the ambience. IEEE Transactions on Haptics 2, 3 (2009), 123-135.

29. Mamykina, L., Mynatt, E., and Terry, M.A. Time Aura: interfaces for pacing. Proc. CHI, ACM (2001), 144-151.

30. Matlin, M.W. Cognition. John Wiley and Sons, NJ, USA, 2008.

31. Microsoft PowerPoint. 2012. http://office.microsoft.com/.

32. Moreno, R. and Mayer, R.E. Cognitive principles of multimedia learning: The role of modality and contiguity. Journal of Educational Psychology 91, 2 (1999), 358-368.

33. Mutewatch. 2012. http://mutewatch.com/.

34. Myers, B.A. Using multiple devices simultaneously for display and control. IEEE Personal Comm. 7, 5 (2000), 62-65.

35. Oakley, I., Kim, Y., Lee, J., and Ryu, J. Determining the feasibility of forearm mounted vibrotactile displays. Proc. VR, IEEE (2006), 27-34.

36. Occhialini, V., Van Essen, H., and Eggen, B. Design and evaluation of an ambient display to support time management during meetings. Proc. INTERACT, Springer-Verlag (2011), 263-280.

37. Paivio, A. Mental representations: a dual coding approach. Oxford University Press, Oxford, England, 1986.

38. Post, L.J., Zompa, I.C., and Chapman, C.E. Perception of vibrotactile stimuli during motor activity in human subjects. Experimental brain research 100, 1 (1994), 107-120.

39. Predebon, J. Time judgments as a function of clock duration: effects of temporal paradigm and an attention-demanding nontemporal task. Percept. Mot. Skills 88, 3 Pt 2 (1999), 1251-4.

40. Spicer, R.P., Lin, Y.R., and Kelliher, A. NextSlidePlease: agile hyperpresentations. Proc. MM, ACM (2009), 1045-1048.

41. Stanley, A.A. and Kuchenbecker, K.J. Evaluation of tactile feedback methods for wrist rotation guidance. IEEE Transactions on Haptics 5, 3 (2012), 240-251.

42. Tam, D. The design and field observation of a haptic notification system for timing awareness during oral presentations. Master's Thesis, University of British Columbia, 2012.

43. Tang, A., McLachlan, P., Lowe, K., Saka, C.R., and MacLean, K. Perceiving ordinal data haptically under workload. Proc. ICMI, ACM (2005), 317-324.

44. Ternes, D. and Maclean, K.E. Designing large sets of haptic icons with rhythm. Proc. EuroHaptics, Springer-Verlag (2008), 199208.

45. Töyssy, S., Raisamo, J., and Raisamo, R. Telling time by vibration. Proc. EuroHaptics, Springer-Verlag (2008), 924-929.

46. Vastenburg, M.H., Keyson, D.V., and Ridder, H. Considerate home notification systems: a field study of acceptability of notifications in the home. Personal Ubiquitous Computing 12, 8 (2008), 555-566.

47. Warnock, D., McGee-Lennon, M.R., and Brewster, S. The impact of unwanted multimodal notifications. Proc. ICMI, ACM (2011), 177-184.

48. Wickens, C.D. Processing resources in attention. Academic Press, NY, USA, 1984, 63-102. 